\title{
Zehn Jahre BPH-Therapie: Evidenz nimmt zu
}

- In den letzten Jahren hat sich die Studienlage zur benignen Prostatahyperplasie (BPH) erheblich verbessert, erläuterte Dr. Volker Moll, Augsburg. 2001 standen zur BPH-Therapie die rasch, aber zeitlich begrenzt wirkenden Alphablocker und der langsam, aber langfristig wirkende 5-AlphaReduktase-Hemmer (5-ARI) Finasterid zur Verfügung. Eine Kombinationstherapie aus 5-ARI und Alphablocker zeigte damals keinen zusätzlichen Benefit in der Symptomlinderung. Das Risiko für Langzeitkomplikationen wie akuten Harnverhalt oder eine BPH-bedingte Operation ließ sich nur unter Finasterid minimieren, zudem war die Lebensqualität noch nicht umfassend untersucht worden.

Heute ist mit der Fixkombination aus Dutasterid und Tamsulosin (Duodart ${ }^{\oplus}$ ) die einzige Behandlungsoption mit Evidenzlevel $1 \mathrm{~b}$ verfügbar. Sie ist im Hinblick auf die Symptomlinderung einer Dutasterid-Monotherapie nach drei Monaten und einer Tamsulosin-Monotherapie nach neun Monaten signifikant überlegen. Sowohl Lebensqualität als auch Patientenzufriedenheit bessern sich gegenüber der jeweiligen Monotherapie ebenfalls signifikant. Zudem ließ sich mit der Kombinationstherapie das Risiko für Langzeitkomplikationen im Vergleich zur Monotherapie mit einem Alphablocker um $66 \%$ senken. Dr. Stephan Madersbacher, Wien, erklärte, dass Alphablocker keinen Effekt auf das Risiko eines Harnverhalts oder einer Operation hätten. „Sie haben in der Therapie von Risiko-Patienten daher nichts mehr verloren", so die Einschätzung von Madersbacher.

„Bei großen Prostaten ist der Vorteil der Kombination am größten", resümierte Prof. Peter Hammerer, Braunschweig. Er empfahl die Kombination bei Patienten mit moderaten bis schweren Symptomen und ab einem Prostatavolumen von $30-40 \mathrm{ml}$. Dies sei auch schon in den EAU- und NICE-Leitlinien berücksichtigt.

Auch in der Therapie des metastasierten Nierenzellkarzinoms (mRCC) finden sich erst seit wenigen Jahren Daten zur Lebensqualität. Prof. Stefan Siemer, Homburg, betonte, dass gerade die Lebensqualität und die Toxizität der Medikamente wichtig für den Patienten seien. Siemer empfahl, vor Behandlungsbeginn mit dem Patienten die möglichen Nebenwirkungen durchzugehen. Er zeigte anhand von Daten aus dem Praxisalltag, wie sich die Nebenwirkungen wie beispielsweise Hypertonie unter einer Pazopanib (Votrient ${ }^{\circledR}$ )-Therapie gut in den Griff bekommen lassen, sodass Patienten von der effektiven Behandlung bei guter Lebensqualität profitieren können. Sebastian Lux

Satellitensymposium "Moderne Therapieansätze bei BPH und Nierenzellkarzinom" im Rahmen des 63. DGU-Kongresses,

Hamburg, 15. September 2011

Veranstalter: GlaxoSmithKline, München

\section{Prostatakarzinom: Abirateron verlängert Gesamtüberleben}

- Der erste steroidale Androgen-Biosynthese-Inhibitor Abirateron (Zytiga ${ }^{\circledR}$ ) blockiert zwei Enzyme (CYP17-17-alpha-Hydroxylase, CYP17-C17,20-Lyase) der AndrogenBiosynthese in Hoden, Nebennieren und Tumorgewebe. Die COU-AA-301-Studie [de Bono JS et al., N Engl J Med 2011, 364: 19952005] hat gezeigt, dass Abirateron das Gesamtüberleben der Patienten signifikant verlängert und Schmerzen sowie skelettale Ereignisse reduziert. Im September 2011 erfolgte die europäische Zulassung zur Therapie des metastasierten kastrationsresistenten Prostatakarzinoms (mCRPC) in Kombination mit Prednison oder Prednisolon bei Versagen einer Docetaxel-basierten Chemotherapie.

In der Phase-III-Studie erhielten 1.195 Patienten mit progredientem $\mathrm{mCRPC}$ nach ein bis zwei Chemotherapien, davon eine Docetaxel-haltige, 2:1 randomisiert Abirateron (1.000 mg/Tag) oder Placebo jeweils plus Prednison/Prednisolon (2-mal $5 \mathrm{mg} / \mathrm{Tag}$ ). Etwa die Hälfte der Patienten war sympto- matisch mit Schmerzen und radiologischer Progression, $90 \%$ hatten Knochenmetastasen. Die Zwischenanalyse zeigte einen signifikanten Überlebensvorteil von knapp vier Monaten für den experimentellen Arm (14,8 versus 10,9 Monate, $p<0,001)$. Alle Subgruppen profitierten gleichermaßen, nur bei Patienten mit schlechtem Performance-Status (ECOG-PS 2) war der Benefit begrenzt.

„Alle sekundären Endpunkte waren kongruent mit dem primären Endpunkt", berichtete Prof. Dr. Kurt Miller, Berlin. Die Zeit bis PSA-Progress (TTPP) war ebenso signifikant verlängert (10,2 versus 6,6 Monate, $p<$ $0,001)$ wie das radiologisch bewertete progressionsfreie Überleben (rPFS, 5,6 versus 3,6 Monate, $p<0,001)$. Die PSA-Ansprechrate erreichte $29,1 \%$ versus $5,5 \%$ in der Kontrollgruppe. „Auch in der Palliation war die Wirkung gut“, so Miller: Der Anteil der Patienten mit Reduktion des Tumorschmerzes war signifikant höher (44\% versus $27 \%$, $\mathrm{p}=0,002$ ) und die Zeit bis zum Auftreten eines skelettalen Ereignisses verdoppelte sich (301 versus 150 Tage, $p=0,0006$ ).

Resultierend aus dem Wirkmechanismus traten unter Abirateron mineralokortikoide Nebenwirkungen wie beispielsweise Flüssigkeitsretention, Hypertonie und Hypokaliämie häufiger auf, die Häufigkeit der meisten unerwünschten Ereignisse lag auf dem Niveau der Kontrollgruppe. Bei der finalen Ergebnisanalyse mit einem Follow-up von median 20,2 Monaten zeigte sich, dass der Überlebensvorteil für die Patienten nochmals zugenommen hatte und sich auf 4,6 Monate steigerte (15,8 Monate versus 11,2 Monate, $\mathrm{p}<0,0001)$. Michael Koczorek

Launch-Pressekonferenz „Markteinführung von Zytiga ${ }^{\oplus}$ (Abirateronacetat): Phase-IIIDaten und klinische Konsequenzen" im Rahmen des 63. DGU-Kongresses, Hamburg, 16. September 2011

Veranstalter: Janssen-Cilag, Neuss 